\title{
Comparison of transmission and the 90-degree holographic recording geometry
}

\author{
Yunping Yang, Ali Adibi, and Demetri Psaltis
}

\begin{abstract}
We compare the system performances of two holographic recording geometries using iron-doped lithium niobate: the 90-degree and transmission geometry. We find that transmission geometry is better because the attainable dynamic range $(M \#)$ is much higher. The only drawback of transmission geometry is the buildup of fanning, particularly during readout. Material solutions that reduce fanning such as doubly-doped photorefractive crystals make transmission geometry the clear winner. (C) 2003 Optical Society of America
\end{abstract}

OCIS codes: $\quad 210.0210,210.2860,090.7330,210.4860,160.2900$.

\section{Introduction}

Many of the large-scale holographic memories demonstrations ${ }^{1-10}$ based on photorefractive crystals $\left(\mathrm{LiNbO}_{3}: \mathrm{Fe}\right)$ were performed by use of the 90-degree geometry, in which the two recording beams interfere inside the crystal by an angle of approximately 90 degrees. ${ }^{3,7,10}$ The grating vector (or the K-vector) in the 90-degree geometry is essentially fixed, while the $\mathrm{K}$-vector in transmission geometry can be varied by changing the outside angle between the two recording beams. Figure 1 shows the schematic setups for the 90-degree and transmission geometries. Previous results suggest that the 90-degree geometry has high angular selectivity and relative insensitivity to holographic scattering and fanning.3,7,10,11 However, the 90-degree geometry has a small dynamic range and recording speed. For example, typical values of the dynamic range measure $(M \#)$ and sensitivity $(S)$ of the 90-degree geometry with lightly iron-doped lithium niobate are $2.0 \mathrm{~cm}^{-1}$ and 0.02 cm J, respectively. ${ }^{11}$ For practical applications, larger values of $M$ \# and $S$ are required (perhaps $M \# \quad 10 \mathrm{~cm}^{-1}$ and $\left.S \quad 1 \mathrm{~cm} \mathrm{~J}\right)$.

One approach to improve the $M$ \# and the sensi-

Y. Yang and D. Psaltis are with the Department of Electrical Engineering, California Institute of Technology, Pasadena, California 91125. A. Adibi is with the Department of Electrical Engineering, Georgia Institute of Technology, Atlanta, Georgia 30332-0250.

Received 26 September 2002; revised manuscript received 26 February 2003.

0003-6935 03 173418-10\$15.00 0

(C) 2003 Optical Society of America tivity for the $\mathrm{LiNbO}_{3}$-based holographic storage system is to increase the doping level. For $\mathrm{LiNbO}_{3}: \mathrm{Fe}$ crystals, the highest practical doping level is approximately 0.06 wt.\% $\mathrm{Fe}_{2} \mathrm{O}_{3}$ and is limited by dark decay. ${ }^{12-14}$ It also has been found that by using $\mathrm{Mn}$ instead of $\mathrm{Fe}$ as a dopant, the practical highest doping level can be increased considerably to obtain a larger $M$ \# and greater sensitivity. ${ }^{15}$ Another way to improve the $M$ \# and sensitivity for the $\mathrm{LiNbO}_{3}$ based holographic storage system is to use transmission geometry instead of the 90-degree geometry. In this paper, we compare the system performances of the 90-degree and the transmission holographic recording geometries by use of iron-doped lithium niobate. The comparison measures are dynamic range $(M$ \#), sensitivity $(S)$, scattering noise, fanning, inter-pixel noise, and capacity.

\section{2. $M$ \# and Sensitivity}

One of the most important system metrics for holographic storage systems is the dynamic range $(M \#)$. When $M$ holograms are multiplexed by using the appropriate recording schedule, ${ }^{16}$ the diffraction efficiency of each hologram $(\eta)$ is given by

$$
\eta={\frac{M \#^{2}}{M}}^{2}
$$

Equation 1 suggests that in multiplexing holograms with a prescribed diffraction efficiency $\eta$, increasing the $M$ \# results in increasing the number of holograms that can be multiplexed $(M)$, thus the capacity of the holographic storage system. On the other hand, with a fixed number of holograms multiplexed, larger $M$ \# results in a higher diffraction efficiency 

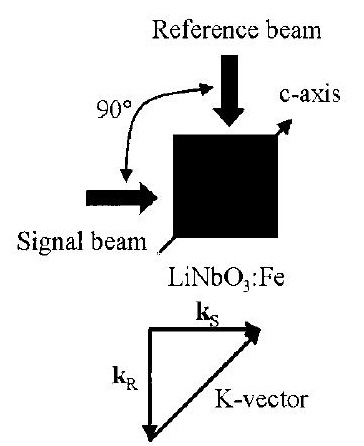

The 90-degree geometry

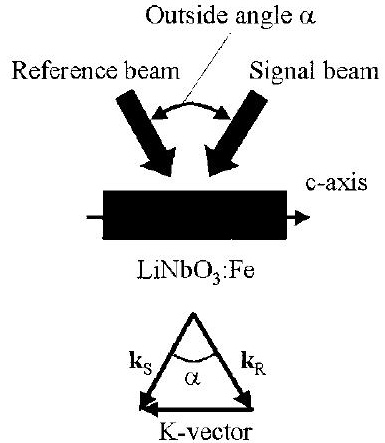

Transmission geometry
Fig. 1. The 90-degree geometry vs. transmission geometry. The $\mathrm{K}$-vector in the 90-degree geometry is almost fixed, while the $\mathrm{K}$-vector in transmission geometry can be varied by changing the outside angle between the two recording beams and is smaller than that of the 90-degree geometry.

for each hologram, therefore a higher signal-to-noise ratio (SNR) and data transfer rate. Another important system metric for a holographic storage system is sensitivity, which determines the recording speed. The larger the sensitivity, the faster we can record the hologram with a fixed recording intensity. For a holographic storage system, it is always desirable to have the largest possible $M$ \# and sensitivity.

Dynamic range $(M \#)$ and sensitivity $(S)$ can be measured by single-hologram recording and erasure experiments. From the single-hologram recording and erasure curve, we can calculate $M$ \# and $S$ using

$$
\begin{aligned}
M \# & =\frac{d \bar{\eta}}{d t}{ }_{t=0} \tau_{\mathrm{e}}, \\
S & =\frac{d \bar{\eta} d t_{t=0}}{I L},
\end{aligned}
$$

where $\tau_{\mathrm{e}}, I$, and $L$ are the erasure time constant, total recording intensity, and the crystal thickness, respectively. In our experiments for measuring $M$ \# and sensitivity, an argon-ion laser beam with a wavelength of $488 \mathrm{~nm}$ was used to record and to erase holograms. The crystal was placed on a rotation stage. The laser beam was split into two equalintensity beams with the intensity of each beam at approximately $10 \mathrm{~mW} \mathrm{~cm}$. The grating vector is always aligned along the $c$-axis. During recording, one beam was blocked from time to time to measure the holographic diffraction efficiency. We used Bragg-mismatched erasure, i.e., during erasure the sample was rotated far away from the Braggmatched position (by at least 50 times that of the selectivity) and illuminated by the same two beams that were used to record the holograms. This guaranteed that the spurious gratings recorded during erasure would have little effect on the measurement of $M$ \# and sensitivity. Moreover, to avoid building strong spurious holograms and fanning, the sample
Table 1. Measured $M$ \# and Sensitivity for the 90-Degree Geometry Crystals

\begin{tabular}{ccccc}
\hline Sample & $\begin{array}{c}\text { Doping level } \\
(\mathrm{mol} \%)\end{array}$ & $\begin{array}{c}\text { Thickness } \\
(\mathrm{mm})\end{array}$ & $M$ \# (per cm) & $S(\mathrm{~cm} \mathrm{~J})$ \\
\hline S1 & 0.01 & 20 & 2.30 & 0.02 \\
S2 & 0.015 & 20 & 2.25 & 0.02 \\
S3 & 0.015 & 20 & 3.34 & 0.03 \\
\hline
\end{tabular}

was rotated 0.02 deg every 10 seconds during erasure. At the end of each period of erasure, the diffraction efficiency was measured by scanning over an adequate range of angle (which covered the Braggmatched position) and by finding the maximum diffraction efficiency with only the reference beam on. Table 1 and Table 2 summarize the measured $M$ \# and sensitivity for three $\mathrm{LiNbO}_{3}: \mathrm{Fe}$ crystals of the 90-degree geometry and three $\mathrm{LiNbO}_{3}: \mathrm{Fe}$ crystals of transmission geometry available in our lab.

From Table 1 and Table 2, we can see that both $M \#$ and sensitivity in transmission geometry are considerably larger than those in the 90-degree geometry. The measured $M$ \#'s of transmission geometry crystals are approximately 10 times of those of the 90-degree geometry crystals. The measured sensitivities of transmission geometry crystals are also approximately 10 times larger than those of the 90-degree geometry crystals. Three factors contribute to the larger $M$ \# and sensitivity in transmission geometry: smaller K-vector, larger effective electrooptic coefficient $r_{\text {eff }}$, and higher average modulation depth. Accordingly, we can represent $M \#$ as the product of three terms corresponding to these three factors:

$$
\begin{aligned}
M \# & =\left(E_{\mathrm{sc}} \tau_{\mathrm{e}} \tau_{\mathrm{r}}\right)\left(n^{3} r_{\mathrm{eff}} 2\right)(m) \\
& =M_{1}(K) M_{2}\left(r_{\mathrm{eff}}\right) M_{3}(m),
\end{aligned}
$$

where $M_{1}(K)=E_{\mathrm{sc}} \tau_{\mathrm{e}} \tau_{\mathrm{r}}$ is a function of the K-vector, $M_{2}\left(r_{\text {eff }}\right)=n^{3} r_{\text {eff }} 2$, and $M_{3}(m)=m$ is the average modulation depth. ${ }^{11}$ In these equations, $E_{\mathrm{sc}}, \tau_{\mathrm{e}}, \tau_{\mathrm{r}}$, and $n$ are the saturation space-charge field for unity modulation depth $(m=1)$, recording time constant, erasure time constant, and refractive index of the crystal, respectively.

Using the first-order approximation, we can analytically solve Kukhtarev equations, which govern

Table 2. Measured $M$ \# and Sensitivity for the Transmission Geometry Crystals $^{a}$

\begin{tabular}{ccccc}
\hline Sample & $\begin{array}{c}\text { Doping level } \\
(\mathrm{mol} \%)\end{array}$ & $\begin{array}{c}\text { Thickness } \\
(\mathrm{mm})\end{array}$ & $M$ \# (per cm) & $S(\mathrm{~cm} \mathrm{~J})$ \\
\hline S4 & 0.01 & 5.0 & 14.46 & 0.22 \\
S5 & 0.015 & 4.5 & 24.01 & 0.14 \\
S6 & 0.03 & 5.0 & 35.72 & 0.34 \\
\hline
\end{tabular}

${ }^{a}$ Extraordinary polarization, outside angle: $22^{\circ}$. 


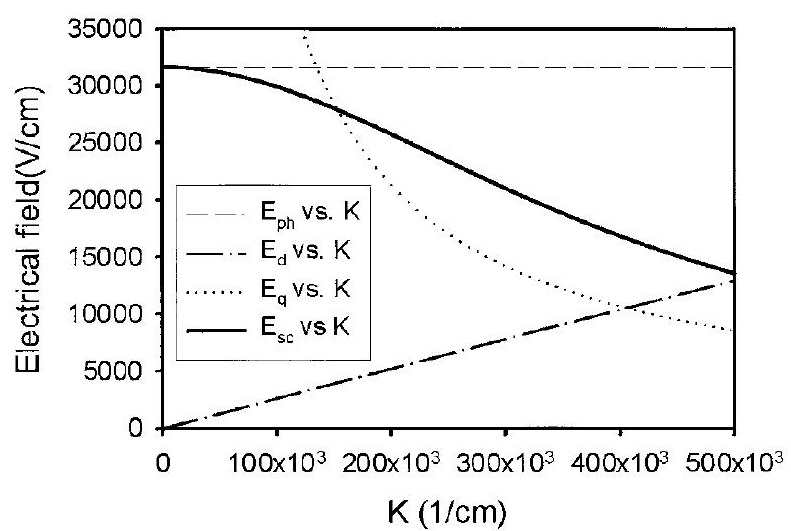

Fig. 2. $\mathrm{E}_{\mathrm{d}}, \mathrm{E}_{\mathrm{q}}, \mathrm{E}_{\mathrm{ph}}$ and $\mathrm{E}_{\mathrm{sc}}$ as functions of the magnitude of the $\mathrm{K}$-vector $(\mathrm{K})$ for a $\mathrm{LiNbO}_{3}$ : Fe crystal. The magnitude of the $\mathrm{K}$-vector in the 90-degree geometry at wavelength of $488 \mathrm{~nm}$ is approximately $427,900 \mathrm{~cm}^{-1}$, while $\mathrm{K}$ of transmission geometry can be varied between 0 to $257,508 \mathrm{~cm}^{-1}$.

the photorefractive effect, to obtain $E_{\mathrm{sc}}$ and $M_{1}(K)$ as the following:

$$
\begin{aligned}
& E_{\mathrm{sc}}=E_{\mathrm{q}} \overline{\frac{E_{\mathrm{ph}}^{2}+E_{\mathrm{d}}^{2}}{\left[\left(N_{\mathrm{A}} N_{\mathrm{D}}\right) E_{\mathrm{ph}}\right]^{2}+\left(E_{\mathrm{d}}+E_{\mathrm{q}}\right)^{2}}}, \\
& M_{1}(K)=E_{\mathrm{q}} \frac{\overline{E_{\mathrm{ph}}{ }^{2}+E_{\mathrm{d}}{ }^{2}}}{E_{\mathrm{d}}+E_{\mathrm{q}}},
\end{aligned}
$$

where $N_{\mathrm{D}}$ and $N_{\mathrm{A}}$ are the total concentrations of the deep (i.e., Fe) traps and the concentration of the ionized deep traps (i.e., $\mathrm{Fe}^{3+}$ ), respectively. ${ }^{17,18}$ In these equations, the saturation field $E_{\mathrm{q}}$, the photovoltaic field $E_{\mathrm{ph}}$, and the diffusion field $E_{\mathrm{d}}$ are given by

$$
\begin{aligned}
E_{\mathrm{q}} & =\frac{e N_{\mathrm{A}}\left(N_{\mathrm{D}}-N_{\mathrm{A}}\right)}{\epsilon K N_{\mathrm{D}}}, \\
E_{\mathrm{ph}} & =\frac{\kappa_{\mathrm{D}} \gamma_{\mathrm{D}} N_{\mathrm{A}}}{e \mu q_{\mathrm{D}} s_{\mathrm{D}}}, \\
E_{\mathrm{d}} & =\frac{k_{\mathrm{B}} T}{e} K,
\end{aligned}
$$

with $\gamma_{\mathrm{D}}, q_{\mathrm{D}} s_{\mathrm{D}}$, and $\kappa_{\mathrm{D}}$ being the recombination rate of electrons in the conduction band, absorption cross section for the excitation of electrons from the deep traps to the conduction band, and the photovoltaic constant of the deep traps (both at the recording wavelength), respectively. Electron charge, Boltzmann constant, and absolute temperature are represented by $e, k_{\mathrm{B}}$, and $T$, respectively. The magnitude of the K-vector is denoted by $K$.

Figure 2 shows $E_{\mathrm{d}}, E_{\mathrm{q}}, E_{\mathrm{ph}}$, and $E_{\mathrm{sc}}$ as functions of $K$ for a $\mathrm{LiNbO}_{3}: \mathrm{Fe}$ with the doping level of 0.015 mol.\% and the oxidation state of $C_{\mathrm{Fe}^{2+}} C_{\mathrm{Fe}^{3+}}=0.03$. The magnitude of the K-vector (i.e., $K$ ) in the 90 degree geometry at the $488-\mathrm{nm}$ wavelength is approximately $427,900 \mathrm{~cm}^{-1}$, while $K$ of transmission

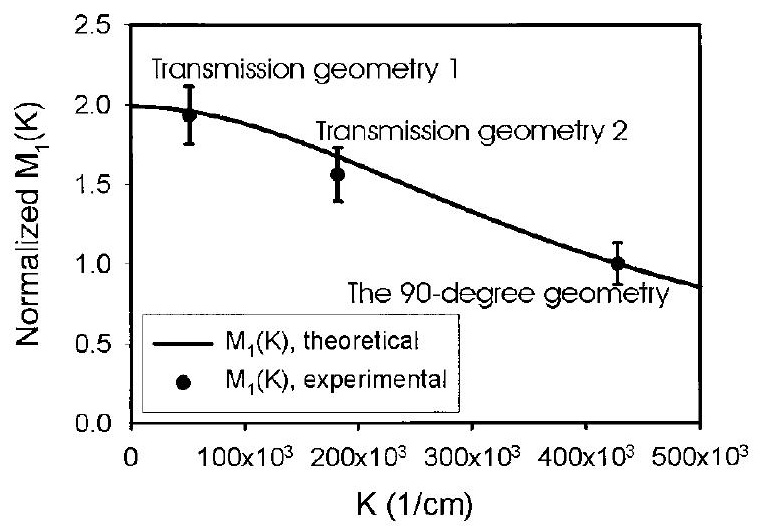

Fig. 3. Theoretical (solid curve) and experimental (filled circles) normalized $\mathrm{M}_{1}(\mathrm{~K})$ for transmission geometry as functions of $\mathrm{K}$ (with the normalized $\mathrm{M}_{1}(\mathrm{~K})$ of the 90 -degree geometry equal to 1 ). For transmission geometry 1 , the smaller $\mathrm{K}$ contributes an increase in the $M \#$ by a factor of 2 compared with the 90 -degree geometry.

geometry can be varied between 0 to $257,508 \mathrm{~cm}^{-1}$ at the same wavelength. For transmission geometry in $\mathrm{LiNbO}_{3}$ (in which $K$ is small) the photovoltaic field $E_{\mathrm{ph}}$ dominates the photorefractive effect, while for the 90-degree geometry (where $K$ is relatively large) the space charge field $E_{\mathrm{sc}}$ is limited by the saturation filed $E_{\mathrm{q}}$. In the crystals we used, the photovoltaic field $E_{\mathrm{ph}}$ (i.e., the dominant field in transmission geometry) is larger than the saturation field of the 90degree geometry. Therefore $M_{1}(K)$ is larger in transmission geometry than that in the 90-degree geometry. Figure 3 shows the theoretical and experimental normalized $M_{1}(K)$ [with the normalized $M_{1}(K)$ of the 90-degree geometry equal to 1$]$ as functions of $K$. From Fig. 3 we can see that for transmission geometry 1 , in which the outside angle between two recording beams is $20^{\circ}$, the smaller $K$ contributes to an increase in the $M$ \# by a factor of 2 compared with the 90-degree geometry.

Owing to the large refractive index of $\mathrm{LiNbO}_{3}(n \approx$ 2.3 for visible light), the angle between two recording beams inside the crystal in transmission geometry is small even when the outside angle is close to $180^{\circ}$. This fact allows us to use both ordinary and extraordinary polarizations in transmission geometry. The electro-optic coefficient of $\mathrm{LiNbO}_{3}$ for extraordinary polarization is three times larger than that for ordinary polarization $\left(r_{33}=3 r_{13}\right)$. In the 90 -degree geometry the angle between two recording beams is approximately $90^{\circ}$. Therefore we can only use ordinary polarization. Because the angle between two beams inside the crystal is small in transmission geometry, the effective electro-optic coefficient for transmission geometry with extraordinary polarization is approximately 3 times that in the 90-degree geometry, which means that $M_{2}\left(r_{\text {eff }}\right)$ of transmission geometry is also approximately 3 times larger than that of the 90-degree geometry. Therefore by using extraordinary polarization in transmission geometry we can improve $M$ \# by a factor of three. 


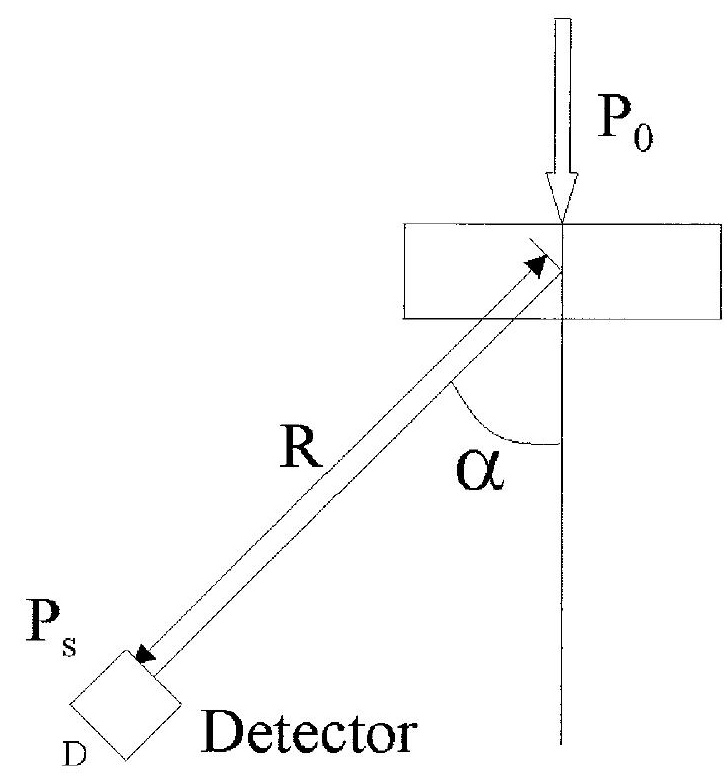

Fig. 4. Experimental setup for measuring scattering as a function of the angle. One beam of plane wave with wavelength $488 \mathrm{~nm}$ and optical power of $\mathrm{P}_{0}$ illuminates the center of the crystal at normal incidence. A detector with aperture diameter $\mathrm{D}$ is placed at a distance $\mathrm{R}$ from the center of the crystal to measure the scattering power $\mathrm{P}_{\mathrm{s}}$.

Another factor that contributes to the larger $M \#$ and sensitivity in transmission geometry is that the local modulation depth in transmission geometry is always close to 1 , which is optimal for holographic recording. However, the local modulation depth in the 90-degree geometry is always less than 1 because of the optical absorption and asymmetry, except for a very small portion of the crystal. In the crystals we used, the average modulation depth of transmission geometry, $M_{3}(m)$, is approximately 2 times larger than that of the 90-degree geometry, which improves the $M \#$ of transmission geometry by another factor of 2 .

From Eqs. (2) and (3) we can see that the only difference between $M$ \# (normalized to thickness $L$ ) and $S$ is that $M$ \# is proportional to the erasure time constant $\tau_{\mathrm{e}}$, while $S$ is inversely proportional to the total intensity of recording $I$. It is known that the erasure time constant $\tau_{\mathrm{e}}$ is inversely proportional to the total intensity, therefore we would expect the same dependence of $S$ on the above three factors as that of $M$ \#. The three factors mentioned previously, i.e., smaller K-vector, larger effective electrooptic coefficient $r_{\text {eff }}$, and higher average modulation depth, contribute to the improvement for sensitivity in the same way as $M$ \# in transmission geometry. This explains the results shown in Table 1 and Table 2.

\section{Scattering Noise}

Noise in holographic storage systems is very important because it determines the overall system performance. In a noise-limited system, lowering the noise level is equivalent to increasing the capacity or obtaining better SNR. One of the main noise

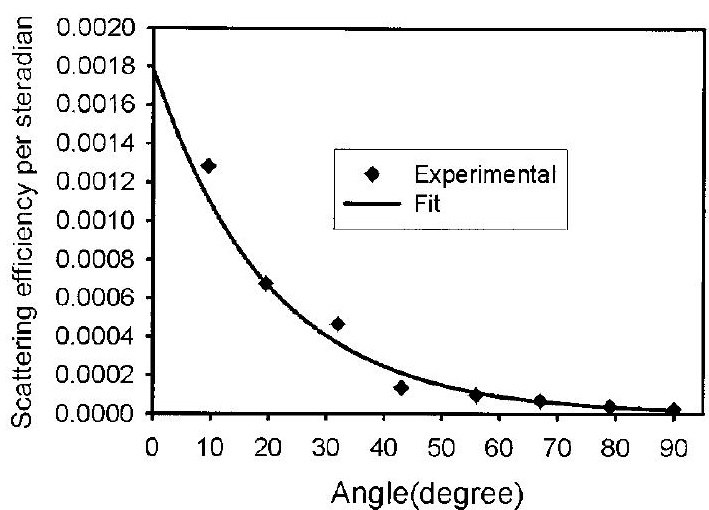

Fig. 5. Measured scattering efficiency per steradian as a function of the angle. The solid line represents an exponential fit to the experimental results. The scattering noise in transmission geometry is larger than that in the 90-degree geometry, especially when the angle between the two recording beams is small.

sources in holographic storage systems is scattering noise. Scattering noise is the result of the scattering of the readout beam by the recording material (i.e., $\mathrm{LiNbO}_{3}$ ). The scattering noise level is a strong function of the angle between the scattering and the scattered beam. Figure 4 shows the experimental setup for measuring scattering of a typical $\mathrm{LiNbO}_{3}: \mathrm{Fe}$ crystal, of which the thickness is $15 \mathrm{~mm}$ and the doping level is $0.015 \mathrm{~mol} \%$. The surfaces of the crystal have been polished to optical quality. One beam of plane wave with a wavelength $488 \mathrm{~nm}$ and an optical power of $P_{0}$ illuminates the center of the crystal at normal incidence. A detector with aperture diameter $D$ is placed at a distance $R$ from the center of the crystal to measure the scattering power $P_{\mathrm{s}}$. The scattering efficiency per steradian $\eta_{\mathrm{s}}$ is calculated as

$$
\eta_{\mathrm{s}}=\frac{\left(P_{\mathrm{s}} P_{0}\right)}{\pi[D(2 R)]^{2}}
$$

Figure 5 shows the measured scattering efficiency per steradian and the exponential fit as a function of the angle. We can see from Fig. 5 that the scattering efficiency decreases exponentially as the scattering angle $\alpha$ changes from 0 to $90^{\circ}$. The scattering noise in transmission geometry (especially when the angle between the two recording beams is very small) is much larger than that in the 90-degree geometry. However, what matters in holographic storage systems is SNR, not the absolute noise level or scattering efficiency. Considering the improvement of $M$ \# in transmission geometry and the fact that the reconstructed signal level is proportional to $(M \#)^{2}$, the signal-to-scattering noise ratio (SSNR) in transmission geometry could be better than that in the 90 degree geometry. Figure 6 shows the normalized signal level, the scattering noise level, and the SSNR in transmission geometry as functions of the angle between the recording beams inside the crystal. The data in all three curves are normalized to the corresponding values in the 90-degree geometry. In other words, the normalized values of the signal level, 

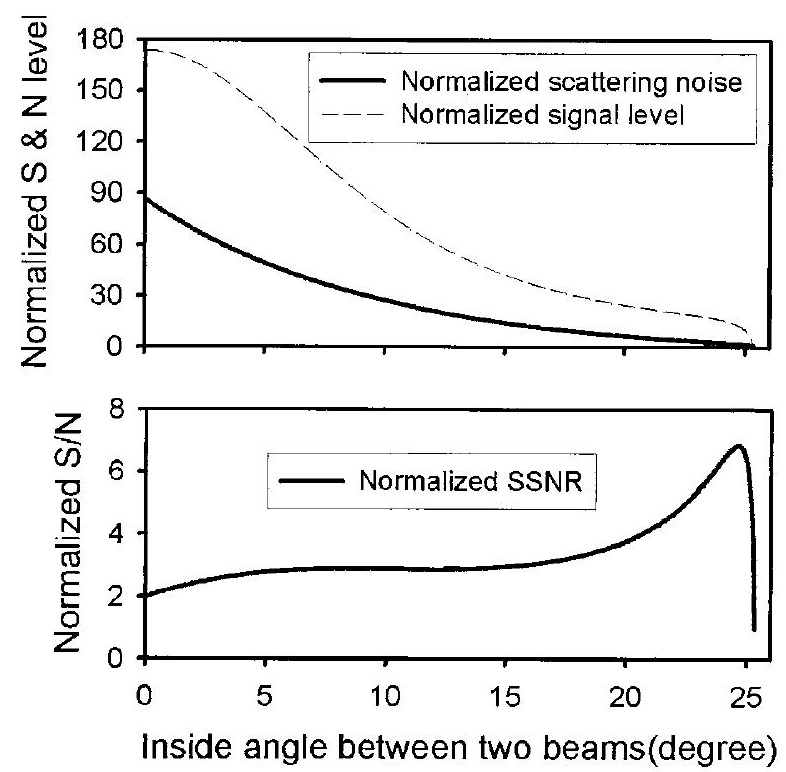

Fig. 6. Normalized signal level, scattering noise, and SSNR in transmission geometry as a function of the angle between the two recording beams inside the crystal with all the corresponding values in the 90-degree geometry normalized to 1 . The SSNR in transmission geometry is better than that in the 90-degree geometry even though the scattering noise level is higher in transmission geometry.

the scattering noise level, and the SSNR for the 90degree geometry are all equal to 1 . In this simulation, we used the doping level of 0.06 wt. $\% \mathrm{Fe}_{2} \mathrm{O}_{3}$ and the thickness of $2 \mathrm{~cm}$ for the $\mathrm{LiNbO}_{3}$ crystal. The results shown in Fig. 6 were calculated by first optimizing the $M \#$ in the 90-degree geometry (through the appropriate choice of the optical absorption, or the oxidation state, at $488 \mathrm{~nm}$ ). Then, the absorption of transmission geometry crystal was chosen to obtain the same sensitivity as that of the 90-degree geometry crystal. By use of this absorption, the $M$ \# and other parameters of transmission geometry crystal were calculated and plotted in Fig. 6. The signal level is proportional to $(M \#)^{2}$. As we can see from Fig. 6, the SSNR in transmission geometry is better than that in the 90-degree geometry even though the scattering noise level is higher in transmission geometry. This is due to the larger $M \#$ in transmission geometry.

Scattering can also occur because of fanning, which builds up during recording or readout. In general, fanning is a more serious problem than static scattering because it is unpredictable, and if allowed to build up for a long time it will eventually lead to complete deterioration of the performance. It is well known that the reason many of the large-scale demonstrations of holographic memory were done in the 90-degree geometry is that fanning is less of a problem. ${ }^{3,7,10}$ Therefore fanning is a very important consideration when we compare the two recording geometries. We performed an experiment to quantitatively assess the fanning behavior. The optical setup is shown in Fig. 7 showing the standard holographic recording geometries for the 90-degree [Fig. 7(a)] and transmission [Fig. 7(b)] geometries. The crystals used were $\mathrm{LiNbO}_{3}: \mathrm{Fe}$ with a doping level $0.015 \mathrm{~mol} . \%$ in both cases. The oxidation state was approximately the same in both cases (measured absorption coefficient at $\lambda=488 \mathrm{~nm}$ was $0.5 \mathrm{~cm}^{-1}$ ). In the first measurement only the reference beam (a

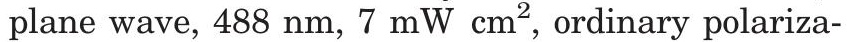
tion, $11^{\circ}$ with respect to the crystal normal for the

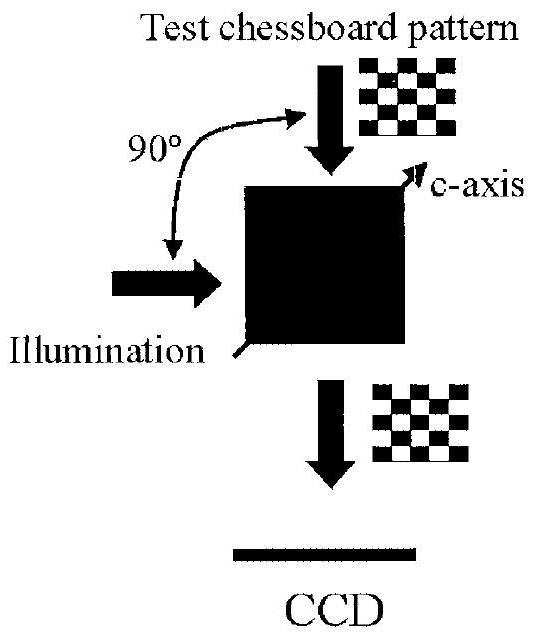

The 90-degree geometry

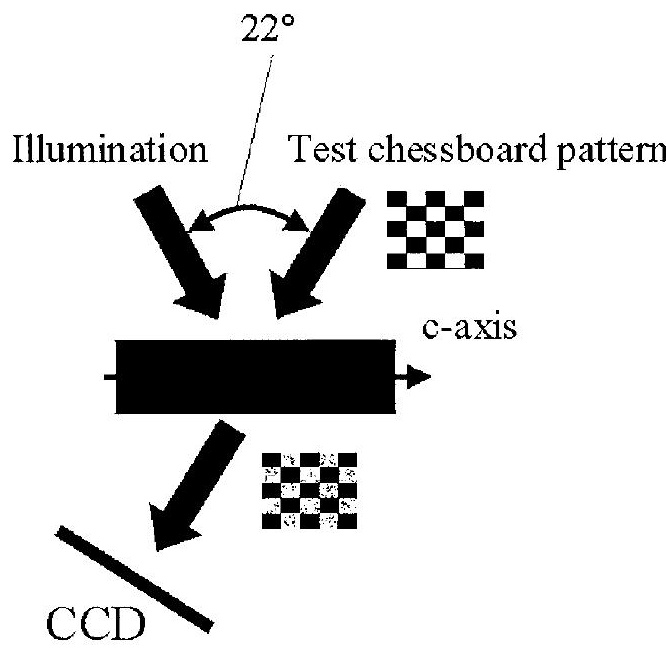

Transmission geometry

(a)

(b)

Fig. 7. Optical setup of holographic recording geometries for (a) the 90-degree and (b) transmission geometries for the measurement of fanning. 


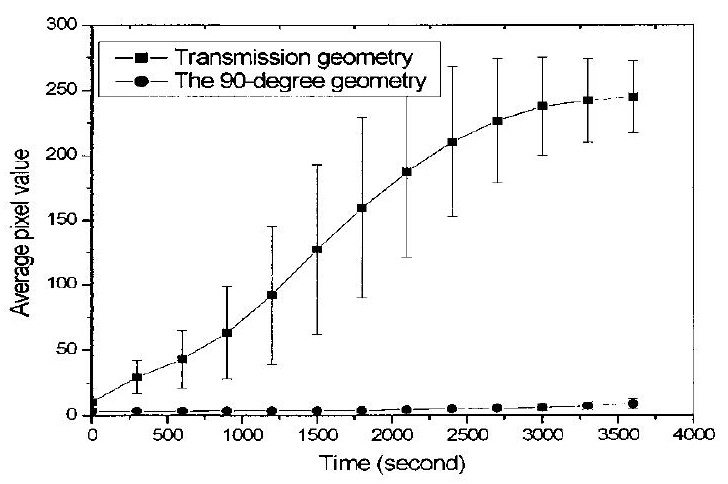

Fig. 8. Averaged pixel value and standard deviation of the CCD signal as a function of time. Within an hour the fanning of transmission geometry grows to a saturation level whereas the 90degree geometry remains almost unaffected after one hour.

transmission case) was present. The light was monitored by a CCD at the plane where we would normally observe the reconstruction of the data page (see Fig. 7). Initially, only the static scattering was picked up by the CCD but as time went on the fanning built up and the CCD signal grew.

In Fig. 8 we plot the averaged pixel value of the CCD signal as a function of time. Also shown in Fig. 8 is the standard deviation of the calculated average signal. Clearly, within an hour the fanning of transmission geometry grows to a saturation level whereas the 90-degree geometry remains almost unaffected after one hour. We assess the impact of the fanning on system performance by introducing a probe beam that is a chessboard pattern that modulates the signal beam. For this measurement we desire to measure the SNR between the probe and the fanning noise as it is read out by the reference beam. We avoid recording a hologram containing the chessboard by using orthogonal polarizations in the signal and reference beams and by minimizing the simultaneous exposure time. The intensity of the probe

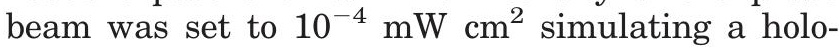

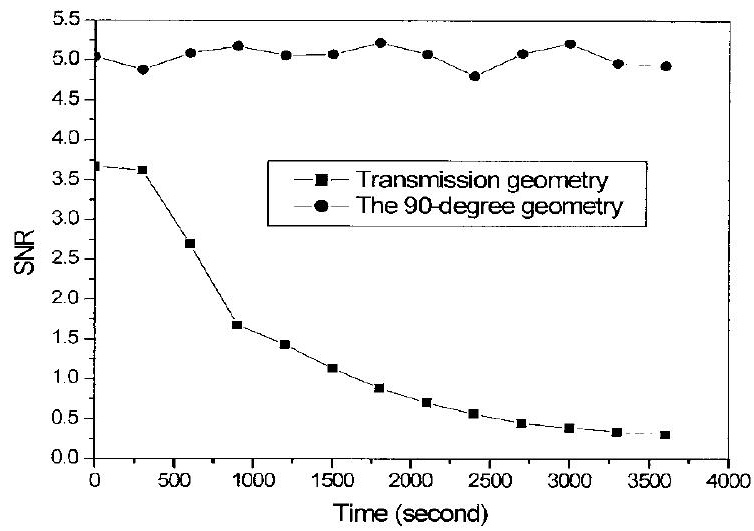

Fig. 9. Measured SNR degradation due to fanning. The SNR remains virtually unchanged for the 90-degree geometry whereas it deteriorates to virtually zero within an hour for transmission geometry. gram with diffraction efficiency equal to $10^{-5}$ illuminated by a beam with $10 \mathrm{~mW} \mathrm{~cm}^{2}$. As shown in Figure 9, the SNR remains virtually unchanged for the 90-degree geometry whereas it deteriorates to virtually zero within an hour for transmission geometry.

From these measurements we see that indeed fanning is the major drawback of transmission geometry. The reduced fanning of the 90-degree geometry in $\mathrm{LiNbO}_{3}: \mathrm{Fe}$ can be attributed partially to the lower sensitivity of this geometry and the large angle between the two recording beams as we already discussed. Fanning depends on the choice of material property as well as the geometry. For instance, fanning is not observed in doubly-doped lithium niobate when it is recorded in the presence of a blue sensitizing beam and read out in the red. ${ }^{19,20}$ This can be attributed to the erasure of the fanning signal by the blue beam during recording and the low sensitivity of the crystal in the red during readout. Fanning is also dramatically reduced in $\mathrm{LiNbO}_{3}: \mathrm{Mn}$ crystals recorded in the blue. ${ }^{15}$

As discussed in the previous section, the sensitivity with extraordinary polarization is larger than that with ordinary polarization. It is known that the buildup of fanning is faster with larger sensitivity. With the same recording time, the buildup of fanning with extraordinary polarization is faster than that with ordinary polarization. Nevertheless, it will take less time with extraordinary polarization to achieve the same diffraction efficiency than that with ordinary polarization, and the fanning will be comparable with both extraordinary and ordinary polarizations during recording. In this sense, the speeds of buildup of fanning with extraordinary and ordinary polarizations in transmission geometry are comparable.

\section{Inter-Pixel Noise}

Another main source of noise in holographic storage systems is inter-pixel noise. Inter-pixel grating is a very important yet largely ignored form of holographic noise. It is caused by rediffraction of the diffracted signal from the gratings formed between the multiple plane-wave (spatial-frequency) components of the signal beam. Inter-pixel grating noise can be considered as crosstalk noise between the pixels (bits) within a page of information, similar to a class of higher-order crosstalk noise in volume holographic interconnection. ${ }^{21,22}$ In the setup of Fourier-plane recording where the holographic recording medium is placed at the Fourier-transform plane of the spatial light modulator (SLM) inter-pixel grating noise occurs directly between the pixels on the SLM. Individual pixels are converted into plane waves inside the storage medium. During holographic recording, these plane waves interfere with the reference beam to form the desired information hologram. In the meantime, they interfere with each other to create inter-pixel noise gratings. Upon readout, the same reference beam is used to reconstruct the plane waves, which are converted back to 


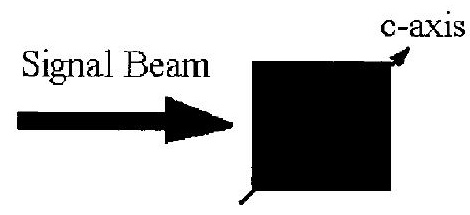

90-degree geometry

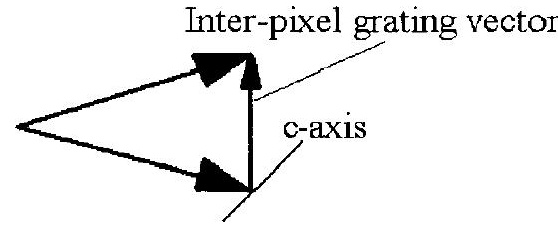

The angle between inter-pixel grating vector and $\mathrm{c}$-axis is 45-degree

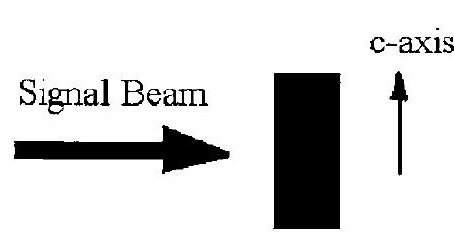

Transmission geometry

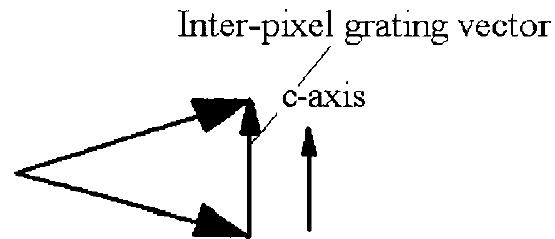

The angle between inter-pixel grating vector and c-axis is 0 -degree

Fig. 10. Inter-pixel grating for the 90-degree geometry and transmission geometry. In transmission geometry, the inter-pixel grating vector is parallel to the $c$-axis of the crystal, while the angle between inter-pixel grating vector and the $c$-axis in the crystal for the 90 -degree geometry is $45^{\circ}$.

the corresponding pixels for data retrieval by imaging optics. In addition, these reconstructed plane waves give rise to secondary diffraction via the inter-pixel gratings, resulting in inter-pixel grating noise. This inter-pixel noise is the main source of noise for largescale holographic memories.

Figure 10 shows the orientations of inter-pixel grating vectors and the $c$-axis in transmission geometry and in the 90-degree geometry. Owing to the symmetry of the SLM pixels and the fact that the spatial frequencies of the pixels are relatively small, the inter-pixel grating vectors in both transmission geometry and the 90-degree geometry can be considered the same and perpendicular to the propagation direction of the signal beam. In transmission geometry, the inter-pixel grating vector is parallel to the $c$-axis of the crystal, while the angle between the inter-pixel grating vector and the $c$-axis of the crystal in the 90-degree geometry is $45^{\circ}$. The grating strength is proportional to the cosine of the angle between the grating vector and the $c$-axis of the crystal, and the diffraction efficiency is proportional to the square of the grating strength. Therefore we would expect the inter-pixel grating noise in transmission geometry to be larger than that in the 90 degree geometry by a factor of 2 .

One approach to monitor the evolution of the interpixel noise gratings is to measure the degradation of the SNR of a testing pattern, e.g., a chessboard, through the crystal during the recording. Figure 11 shows the experimental setup for measuring the evolution of inter-pixel noise gratings inside the crystal. The SLM, which is illuminated by a plane wave, is imaged to the CCD plane by a $4-f$ system consisting of two lenses. The crystal is placed at the Fouriertransform plane of the SLM. Each pixel of the SLM generates a plane wave with a different spatial frequency through the crystal. These plane waves interfere with each other to form the inter-pixel noise gratings. Figure 12 shows the measured SNR degradation due to inter-pixel noise as a function of time for one of the 90-degree geometry and one of transmission geometry $\mathrm{LiNbO}_{3}: \mathrm{Fe}$ crystals due to interpixel noise. The doping levels of both the 90-degree and transmission geometry crystals are the same: $0.015 \mathrm{~mol} \% \mathrm{Fe}$. Also, the absorption coefficients of these two crystals at $488 \mathrm{~nm}$ are almost the same, 0.5 $\mathrm{cm}^{-1}$, which means that both crystals have approximately the same oxidation states. The interaction

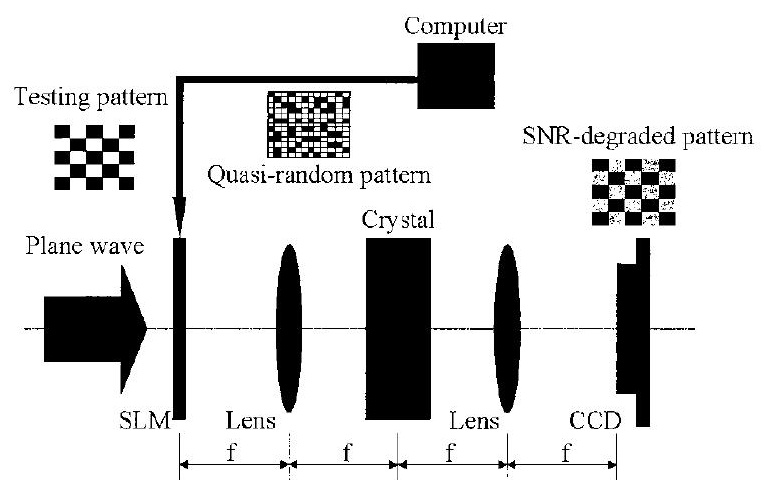

Fig. 11. Experimental setup for monitoring the evolution of the inter-pixel noise grating. The SLM, which is illuminated by a plane wave, is imaged to the CCD plane by a 4- $f$ system consisting of two lenses. The crystal is placed at the Fourier-transform plane of the SLM. 


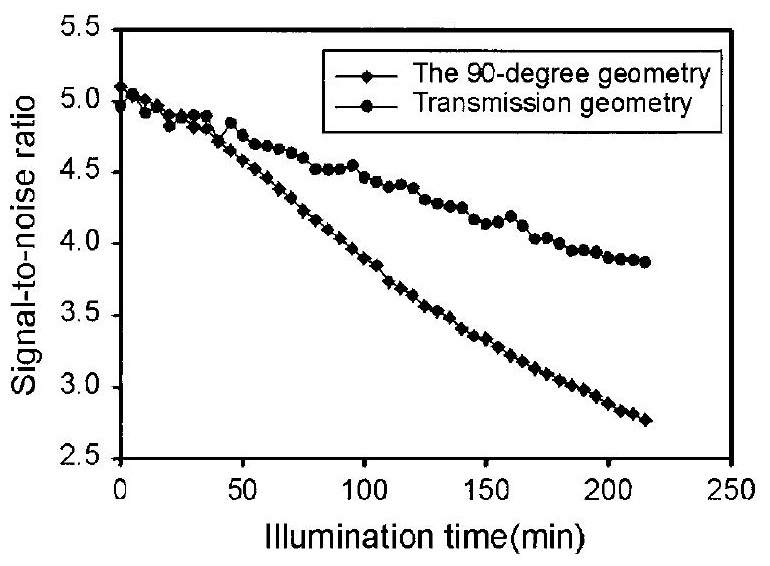

Fig. 12. Measured SNR degradation due to inter-pixel noise as a function of time for one of the 90-degree geometry and one of transmission geometry $\mathrm{LiNbO}_{3}: \mathrm{Fe}$ crystals due to inter-pixel noise. The interaction length of the 90-degree geometry crystal is $20 \mathrm{~mm}$, while that of transmission geometry crystal is $4.5 \mathrm{~mm}$.

length of the 90-degree geometry crystal is $20 \mathrm{~mm}$ while that of transmission geometry crystal is 4.5 $\mathrm{mm}$. The intensity of the plane wave that illuminates the SLM for both crystals is the same. To obtain the results shown in Fig. 12, quasi-random patterns were imprinted to the SLM by a computer. From time to time, a big chessboard pattern was used to monitor the degradation of the SNR of the image after the crystal. Note that in this experiment, we illuminate the crystal with only the signal beam. We changed the quasi-random pattern every 10 seconds to simulate the actual recording process and to avoid the buildup of holographic scattering noise. Therefore we expect that any degradation in the SNR (shown in Fig. 12) of a single image going through the crystal is due to the inter-pixel noise. The SNR is calculated as:

$$
S N R=\frac{\mu_{1}-\mu_{0}}{{\overline{\sigma_{1}}}^{2}+\sigma_{0}^{2}},
$$

where $\mu_{1}, \sigma_{1}, \mu_{0}$, and $\sigma_{0}$ are the average pixel value of ON pixels, the standard derivation of the pixel value of $\mathrm{ON}$ pixels, the average pixel value of the $\mathrm{OFF}$ pixels, and the standard derivation of the pixel value of the OFF pixels, respectively. From Fig. 12 we can see that the speed of degradation of the SNR in the 90-degree geometry crystal is 2-times larger than that in the transmission geometry crystal. The inter-pixel noise intensity is proportional to $L^{2}[\exp (-\alpha L)]^{2}$ with $\alpha$ and $L$ being the intensity absorption coefficient and the thickness of the crystal, respectively. The oxidation state of the 90-degree geometry crystal was optimized to obtain the best $M$ \#. The optimum absorption coefficient for the 90 degree geometry crystal with thickness $L=2 \mathrm{~cm}$ is $\alpha=1 L=0.5 \mathrm{~cm}^{-1}$. The absorption coefficient of transmission geometry crystal was very close to that of the 90-degree geometry crystal (owing to similar doping concentration and similar annealing treat- ment). Therefore we expect the ratio of the interpixel noise level $\left(N_{\text {IP }}\right)$ in the two cases to be

$$
\begin{aligned}
\frac{N_{\text {IP 90-deg }}}{N_{\text {IP Trans }}} & =\cos ^{2}\left(45^{\circ}\right) \frac{(2)^{2} \exp (-0.5 \times 2 \times 2)}{(0.45)^{2} \exp (-0.5 \times 0.45 \times 2)} \\
& =2.1
\end{aligned}
$$

which is in good agreement with the results shown in Fig. 12. Therefore the apparent worse inter-pixel noise in the 90-degree geometry is due to the thickness of the crystal. If we use the same thickness for the crystals used in the two geometries, we will obtain

$$
\frac{N_{\text {IP 90-deg }}}{N_{\text {IP Trans }}}=\cos ^{2}\left(45^{\circ}\right)=12 .
$$

Our results suggest that under the same conditions (same doping level, same thickness, etc.), the interpixel noise level in transmission geometry is twice that in the 90-degree geometry. The comparison of the measure of signal to inter-pixel noise ratio, which is more important for holographic storage systems, must be performed with precautions. If we record the same number of holograms in both geometries, the diffracted signal level of each hologram is much larger in transmission geometry owing to the larger $M$ \#. This gives transmission geometry a better inter-pixel signal to noise performance. However, if we record as many holograms as possible in both geometries to obtain the highest capacity with the minimum acceptable diffraction efficiency, the signal to inter-pixel noise performance of the 90 -degree geometry becomes better than that of transmission geometry. This is because the signal levels in both cases are the same while the noise level in the 90degree geometry is smaller. The longer recording time of transmission geometry (due to recording more holograms) will increase the inter-pixel noise level further. For a case between the two extremes discussed, either geometry can have better signal to inter-pixel noise performance depending on the number of holograms that are recorded. If we are not very close to the two extremes, we expect the interpixel noise performance in the 90-degree geometry and in transmission geometry to be comparable.

\section{Capacity}

Capacity is a key performance measure in holographic storage systems. One of the advantages of transmission geometry is that we can use extraordinary polarization for both the recording and the readout beam. By switching to extraordinary polarization from ordinary polarization, we increase the $M$ \# (and therefore, the number of multiplexed holograms) by a factor of approximately 3 . The angular selectivities in transmission geometry with both polarizations are almost equal, which means that using extraordinary polarization will not sacrifice the capacity in transmission geometry. This is shown in Fig. 13, which depicts the experimentally measured and theoretically calculated angular selec- 


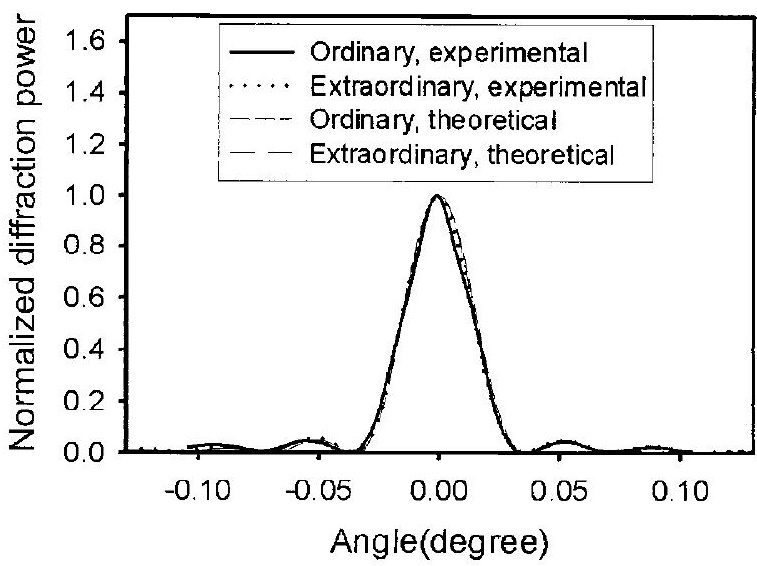

Fig. 13. Experimentally measured and theoretically calculated angular selectivities for one transmission geometry crystal with both extraordinary and ordinary polarizations. No apparent difference between the angular selectivities of the two cases is seen.

tivity curves for one transmission geometry $\mathrm{LiNbO}_{3}: \mathrm{Fe}$ crystal with extraordinary and ordinary polarizations. is

The angular selectivity of the 90-degree geometry

$$
\Delta \theta=\lambda L,
$$

while the angular selectivity of transmission geometry is

$$
\Delta \theta=\frac{\lambda \cos \left(\theta_{\mathrm{s}}\right)}{L \sin \left(\theta_{\mathrm{s}}+\theta_{\mathrm{r}}\right)},
$$

where $\lambda$ is the reading wavelength outside the crystal; $L$ is the interaction length; and $\theta_{\mathrm{s}}$ and $\theta_{\mathrm{r}}$ are the incident angles (inside the crystal) of the signal and the reference beams, respectively. With the same interaction length of the crystal, the angular selectivity of the 90-degree geometry is better than that of transmission geometry. This may suggest that more holograms can be angularly multiplexed by using the 90-degree geometry, and that the 90-degree geometry has a larger capacity than transmission geometry. However, this conclusion is based on the assumption that the holographic system has enough dynamic range to record as many holograms as the angular selectivity allows. In reality, the number of holograms that can be multiplexed in a $\mathrm{LiNbO}_{3}$ crystal is limited by the dynamic range $(M \#)$ and not by the angular selectivity. As a quantitative example, consider a $1 \mathrm{~cm} \times 1 \mathrm{~cm} \times 1 \mathrm{~cm} \mathrm{LiNbO}$ crystal with $M \#=10$ for recording wavelength $\lambda=532 \mathrm{~nm}$. Also, assume that the minimum acceptable diffraction efficiency of each hologram is $\eta_{\min }=10^{-4}$. This allows the multiplexing of $M=1000$ in the crystal. If we use angular multiplexing and we allow a range of 40 degree for the variation of the angle of the reference beam outside the crystal, we need to put the reference beams of the different holograms 40 $1000=0.04^{\circ}$ apart. The angular selectivity of the crystal for the 90-degree geometry is $532 \mathrm{~nm} 1 \mathrm{~cm}=$
$5.32 \times 10^{-5}$ radians $=0.003^{\circ}$. This selectivity allows for the recording of much more than 1000 holograms allowed by the limited $M$ \#. Therefore, with current values of $M \#$, angular selectivity is not a limiting factor in angular multiplexing of holograms in $\mathrm{LiNbO}_{3}$ crystals. Therefore for dynamic range limited holographic storage systems, transmission geometry offers a higher storage capacity than the 90-degree geometry.

\section{Discussion}

The results presented in the previous sections suggest that transmission geometry is a better choice for holographic storage mainly due to its higher dynamic range $(M \#)$ and sensitivity. The main advantages of the 90-degree geometry are insensitivity to holographic scattering and fanning $3,7,10$ as well as the possibility of designing compact memory modules. ${ }^{23}$

Strong fanning in sensitive singly-doped $\mathrm{LiNbO}_{3}$ crystals has been a major obstacle in the implementation of holographic memories in transmission geometry. All the read-write memory systems demonstrated to date use the 90-degree geometry to avoid fanning, thereby sacrificing both $M \#$ and $S$. The holograms recorded by using both geometries are not persistent, i.e., they are erased during readout. Recently, two-center recording has been proposed as a method for recording persistent holograms in doubly-doped $\mathrm{LiNbO}_{3}$ crystals. ${ }^{19,20}$ Two-center recording is a special case of gated holographic recording ${ }^{24-26}$ in which a hologram is recorded by two recording beams only when a sensitizing (or gating) beam is present. The hologram is read by one of the recording beams with the sensitizing beam absent. Therefore the holograms are not erased during readout. In addition to persistence, gated holographic recording reduces the fanning in holographic recording considerably. Holographic scattering and fanning are highly suppressed in two-center recording both during the recording phase (due to the presence of the sensitizing beam) and during the reading phase (due to the insensitivity of the crystal to the reading wavelength in the absence of the sensitizing beam). Therefore the advantage of the 90-degree geometry over transmission geometry disappears in two-center recording.

Another advantage of the 90-degree geometry is that the reference beam and the signal beam propagate in two perpendicular directions. This allows the design of compact architectures for the holographic memory module. ${ }^{23}$ It also makes the implementation of the phase-conjugate readout much easier compared to transmission geometry. ${ }^{27,28}$

The choice of the recording geometry involves a trade-off between the larger capacity and sensitivity on the one hand and the architecture design on the other hand. If maximum capacity and speed is the first priority, transmission geometry with more sophisticated architecture must be chosen. However, for the compact low-cost holographic memory module, the 90-degree geometry is a better choice at the expense of reduction in capacity and speed. ${ }^{23}$ 


\section{Conclusions}

The system performances of two holographic recording geometries (the 90-degree and transmission geometries) with iron-doped lithium niobate were compared here. The comparison was based on dynamic range $(M \#)$, sensitivity, scattering noise, fanning, inter-pixel noise, and capacity. The $M$ \# and sensitivity are larger in transmission geometry than those in the 90-degree geometry. The measured $M \#$ and sensitivity of transmission geometry are 10 times larger than those of the 90-degree geometry for $\mathrm{LiNbO}_{3}: \mathrm{Fe}$ crystals with almost the same doping levels and oxidation states as are available in our lab. Three factors contribute to the larger $M$ \# and sensitivity in transmission geometry: smaller K-vector, larger effective electro-optic coefficient $r_{\text {eff }}$, and higher average modulation depth. Although the scattering noise level in transmission geometry is larger, considering the remarkable gain in the $M$ \#, the signal to scattering noise ratio (SSNR) is better in transmission geometry than that in 90-degree geometry. The inter-pixel noises of the two recording geometries are comparable. Although the angular selectivity in the 90-degree geometry is higher for a dynamic range limited holographic storage system, transmission geometry has a higher capacity than the 90-degree geometry. Finally, fanning is stronger in transmission geometry when singly doped $\mathrm{LiNbO}_{3}: \mathrm{Fe}$ crystals are used.

This effort was sponsored by NSF, Center for Neuromorphic Systems Engineering, Engineering Research Center, Defense Advanced Research Projects Agency.

\section{References}

1. F. Mok, M. Tackitt, and H. Stoll, "Storage of 500 highresolution holograms in a $\mathrm{LiNbO}_{3}$ crystal," Opt. Lett. 8, 605607 (1991).

2. G. Rakujic, V. Levya, and A. Yariv, "Optical data storage by using wavelength multiplexed volume holograms," Opt. Lett. 17, 1471-1473 (1992).

3. F. Mok, "Angle-multiplexed storage of 5,000 holograms in lithium niobate," Opt. Lett. 18, 915-917 (1993).

4. J. F. Heanue, M. C. Bashaw, and L. Hesselink, "Volume holographic storage and retrieval of digital data," Science 265, 749-752 (1994).

5. D. Psaltis and F. Mok, "Holographic memories," Sci. Am. 273, 70-76 (1995).

6. J. H. Hong, I. McMichael, T. Y. Chang, W. Christian, and E. G. Paek, "Volume holographic memory system: techniques and architectures," Opt. Eng. 34, 2193-2203 (1995).

7. G. Burr, F. Mok, and D. Psaltis, "Angle and space multiplexed holographic storage using the 90-degree geometry," Opt. Commun. 117, 49-55 (1995)

8. I. McMichael, W. Christian, D. Pletcher, T. Y. Chang, and J. H. Hong, "Compact holographic storage demonstrator with rapid access,” Appl. Opt. 35, 2375-2379 (1996).
9. R. M. Shelby, J. A. Hoffnagle, G. W. Burr, C. M. Jefferson, M.-P. Bernal, H. Coufal, R. K. Grygier, H. Günther, R. M. Macfarlane, and G. T. Sincerbox, "Pixel-matched holographic data storage with megabit pages," Opt. Lett. 22, 1509-1511 (1997).

10. X. An, D. Psaltis, and G. Burr, "Thermal fixing of 10,000 holograms in $\mathrm{LiNbO}_{3} \mathrm{Fe}$," Appl. Opt. 38, 386-393 (1999).

11. G. Burr, "Volume holographic storage using the $90^{\circ}$ geometry," Ph.D dissertation, California Institute of Technology, Pasadena, Calif. (1996).

12. K. Peithmann, A. Wiebrock, and K. Buse, "Photorefractive properties of highly-doped lithium niobate crystals in the visible and near-infrared," Appl. Phys. B 68, 777-784 (1999).

13. I. Nee, M. Müller, K. Buse, and E. Krätzig, "Role of iron in lithium-niobate crystals for the dark-storage time of holograms," J. Appl. Phys. 88, 4282-4286 (2000).

14. Y. Yang, I. Nee, K. Buse, and D. Psaltis, "Ionic and electronic dark decay of holograms in $\mathrm{LiNbO}_{3}: \mathrm{Fe}$ crystals," Appl. Phys. Lett. 78, 4076-4078 (2001).

15. Y. Yang, K. Buse, and D. Psaltis, "Photorefractive recording in $\mathrm{LiNbO}_{3}: \mathrm{Mn}$," Opt. Lett. 27, 158-160 (2002).

16. D. Psaltis, D. Brady, and K. Wagner, "Adaptive optical networks using photorefractive crystals," Appl. Opt. 27, 17521759 (1988).

17. N. V. Kukhtarev, V. B. Markov, S. G. Odulov, M. S. Soskin, and V. L. Vintekii, "Holographic storage in electrooptic crystals, 1. steady-state," Ferroelectrics 22, 949-960 (1979).

18. P. Yeh, Introduction to Photorefractive Nonlinear Optics (Wiley, New York, 1993).

19. K. Buse, A. Adibi, and D. Psaltis, "Non-volatile holographic storage in doubly doped lithium niobate crystals," Nature 393, 665-668 (1998).

20. A. Adibi, K. Buse, and D. Psaltis, "Two-center holographic recording," J. Opt. Soc. Am. B 18, 584-601 (2001).

21. X. Yi, P. Yeh, and C. Gu, "Statistical-analysis of cross-talk noise and storage capacity in volume holographic memory," Opt. Lett. 19, 1580-1582 (1994).

22. M. P. Bernal, G. W. Burr, H. Coufal, and M. Quintanilla, "Balancing interpixel cross talk and detector noise to optimize areal density in holographic storage systems," Appl. Opt. 37, 5377-5385 (1998).

23. J.-J. P. Drolet, E. Chuang, G. Barbastathis, and D. Psaltis, "Compact, integrated dynamic holographic memory with refreshed holograms," Opt. Lett. 22, 552-554 (1997).

24. D. von der Linde, A. M. Glass, and K. F. Rodgers, "Multiphoton photorefractive processes for optical storage in $\mathrm{LiNbO}_{3}$," Appl. Phys. Lett. 25, 155-157 (1974).

25. H. Guenther, R. Macfarlane, Y. Furukawa, K. Kitamura, and R. Neurgaonkar, "Two-color holography in reduced nearstoichiometric lithium niobate," Appl. Opt. 37, 7611-7623 (1998).

26. L. Hesselink, S. S. Orlov, A. Liu, A. Akella, D. Lande, and R. R. Neugaonkar, "Photorefractive materials for nonvolatile volume holographic data storage," Science 282, 1089-1094 (1998).

27. W. Liu and D. Psaltis, "Pixel size limit in holographic memories," Opt. Lett. 24, 1340-1342 (1999).

28. G. W. Burr and I. Leyva, "Multiplexed phase-conjugate holographic data storage with a buffer hologram," Opt. Lett. 25, 499-501 (2000). 